UTTG-21-97

hep-th/9706226

June 30, 1997

\title{
F-theory, SO(32) and Toric Geometry
}

\author{
Philip CANDELAS'] \\ and \\ Harald SKARKE\# \\ Theory Group \\ Department of Physics \\ University of Texas at Austin \\ Austin, TX 78712, USA
}

\begin{abstract}
We show that the F-theory dual of the heterotic string with unbroken $\operatorname{Spin}(32) / \mathbb{Z}_{2}$ symmetry in eight dimensions can be described in terms of the same polyhedron that can also encode unbroken $E_{8} \times E_{8}$ symmetry. By considering particular compactifications with this $K 3$ surface as a fiber, we can reproduce the recently found 'record gauge group' in six dimensions and obtain a new 'record gauge group' in four dimensions. Our observations relate to the toric diagram for the intersection of components of degenerate fibers and our definition of these objects, which we call 'tops', is more general than an earlier definition by Candelas and Font.
\end{abstract}

\footnotetext{
*e-mail: candelas@physics.utexas.edu

\# e-mail: skarke@zerbina.ph.utexas.edu
} 


\section{Introduction}

F-theory [1] was originally defined as a particular D-manifold vacuum of the type IIB string. Alternatively, it may be seen as a decompactification of the type IIA string, thus lifting the well known duality between the type IIA string and the heterotic string in 6 dimensions [2, 3] to a duality between the heterotic string on $T^{2}$ and F-theory on an elliptically fibered $K 3$ surface. This has afforded new insights into the study of compactifications of the heterotic string [4, 5]. The mechanisms by which enhanced gauge groups arise are quite different on the two sides of the duality: On the F-theory side, as for the type IIA string, singularities of the manifold give rise to gauge groups; while on the heterotic side, singularities of the manifold give rise to gauge group enhancement only if small instantons of the gauge bundle lie precisely on these singularities [6, 7]. In ref. [8] several methods for constructing the relevant bundles were given. These results were recently used [9] to derive precisely which gauge groups and how many tensor multiplets occur when $k$ instantons are placed on a singularity of type $G$, with $G$ any simply laced Lie group.円

Practically all Calabi-Yau manifolds studied by physicists have an interpretation in terms of toric geometry, so it is natural to consider the manifestations of the above phenomena also in these terms. Whereas earlier studies in this context [13, 14] focused on local properties, perhaps the most elegant application involves considering complete polyhedra: Take an elliptic Calabi-Yau threefold that has $K 3$ fibers. In this case it turns out that the $K 3$ polyhedron is contained, as a subpolyhedron, in the Calabi-Yau polyhedron, and that the Dynkin diagrams of the gauge groups that occur upon compactification of the type IIA string on the corresponding threefold [15] can be seen in this $K 3$ polyhedron. This phenomenon, which can be explained in terms of the intersection patterns of the toric divisors [16], can of course be lifted to F-theory and has been studied extensively in this context [17. So far, however, these studies have focused on the F-theory or IIA duals of compactifications of the $E_{8} \times E_{8}$ string. The purpose of the present note is to show that toric methods can equally easily be used for the description of the duals of the $S O(32)$ string. In fact, we will find that the same three-dimensional polyhedron that leads to a $K 3$ surface with two $E_{8}$ singularities may also (upon choosing a different elliptic fibration and blowing down a different set of toric divisors) lead to a $D_{16}$ singularity. This is what we will explain in section two. In

\footnotetext{
${ }^{1}$ Similar results had been derived with different methods in 10, 11, 12.
} 
section three we consider two examples of compactifications to lower dimensions, thereby reproducing the recently found [9] 'record gauge group' in six dimensions and obtaining a new 'record gauge group' in four dimensions.

\section{Eight dimensional vacua}

An eight dimensional F-theory vacuum is determined by an elliptically fibered $K 3$ surface with a section, where all components of the exceptional fibers except for those intersecting the section are blown down [5]. The gauge group is determined by the $A D E$ classification of quotient singularities of surfaces. The intersection matrix of the blown-down divisors is given by minus the Cartan matrix of the corresponding Lie algebra.

Many $K 3$ surfaces can be constructed as hypersurfaces in toric varieties, in the following way [19]: Consider a pair of reflexive the dimensional polyhedra $\left(\Delta, \Delta^{*}\right)$ (for example, $\Delta$ could be the Newton polyhedron of a weighted projective space), with vertices in a pair of dual lattices $(M, N)$. Then each point in $\Delta^{*}$, except for the origin $\mathbf{0}$, determines a ray $v_{i}$ in $N$. Each of these rays corresponds [18 to a homogeneous coordinate $z_{i}$ of the ambient space $\mathbb{P}_{\Delta^{*}}$ and, consequently, to a divisor $D_{i}=\left\{z_{i}=0\right\}$. The $K 3$ surface is determined by a divisor in the class $\left[\sum_{i} D_{i}\right]$. A divisor in $\mathbb{P}_{\Delta^{*}}$ that does not correspond to a point interior to a facet (a codimension one face) of $\Delta^{*}$ results upon intersection with the $K 3$ surface in a divisor in the $K 3$ surface. Therefore the intersection pattern of divisors in the $K 3$ surface can be determined by calculating intersections of the type

$$
D_{i} \cdot D_{j} \cdot \sum_{k} D_{k}
$$

in $\mathbb{P}_{\Delta^{*}}$. This calculation has been considered in detail in [16] leading to the following simple rules: Mutual intersections of divisors in the $K 3$ surface are nonzero if and only if the corresponding points are joined by an edge $\theta^{*}$ of $\Delta^{*}$; in this case their intersection number is the length $l(\theta)$ of the dual edge $\theta$ of $\Delta$ ( $\theta$ has length $l$ if it has $l-1$ interior lattice points). Self-intersections of divisors interior to $\theta^{*}$ are $-2 l$; the corresponding divisors are sums of $l$ rational curves. The self-intersection of a divisor corresponding to a vertex depends on the

\footnotetext{
${ }^{2}$ Our notation is standard. For a definition of reflexivity and an introduction to toric geometry written for physicists see, for example, [20].
} 
geometry of $\Delta^{*}$ in a more sophisticated way. It is 0 , however, in the case of a regular fiber and -2 for any component of a degenerate fiber of an elliptic fibration.

These results were used to explain the observation made in [15] that the extended Dynkin diagrams of enhanced gauge groups are visible in the toric polyhedron. As noted in [15] and explained in [21], the fact that a $K 3$ surface is an elliptic fibration manifests itself torically in the fact that $\Delta^{*}$ contains the polygon corresponding to the generic fiber as a reflexive subpolyhedron.

In this section we will consider a particular family of $K 3$ surfaces which, by the results of [15], can give rise to the group $E_{8} \times E_{8}$; it is the mirror family to the one determined by hypersurfaces of degree 12 in $\mathbb{P}_{1,1,4,6}$. The principal point that we make in this paper is that the same dual pair of polyhedra can also give rise to the gauge group $S O(32)$. In suitable coordinates, the dual polyhedron $\Delta^{*}$ is the convex hull of the points

$$
(1,0,0), \quad(0,1,0), \quad(-2,-3,6), \quad(-2,-3,-6)
$$

Altogether $\Delta^{*}$ contains 39 lattice points. By the results of [22, 23] there is no three dimensional reflexive polyhedron with a larger number of lattice points (there is precisely one other polyhedron with the same number of points, namely the Newton polyhedron of the degree 6 surface in $\left.\mathbb{P}_{1,1,1,3}\right)$. Of these points 21 are 'relevant' in the sense that they are not interior to facets. As there are three independent relations of linear equivalence among the divisors in $\mathbb{P}_{\Delta^{*}}$ we see that the Picard lattice of a generic member of the corresponding family of $K 3$ surfaces has rank 18. Figure 1 consists of a picture of this polyhedron, showing that it contains two different reflexive triangles. We have omitted the 'irrelevant' points (the points interior to facets) except those belonging to the reflexive triangles.

In the first case this is the well known 'Weierstrass triangle', the convex hull of the points

$$
(1,0,0), \quad(0,1,0), \quad \text { and } \quad(-2,-3,0)
$$

lying in the plane $x_{3}=0$. The generic fiber is determined by a Weierstrass equation in the coordinates corresponding to the vertices of this triangle, with coefficients that depend on the coordinates coming from the points outside the plane $x_{3}=0$. This triangle divides $\Delta^{*}$ into two halves, each of which is an ' $E_{8}$ top' as introduced in [15]. The section of the fibration can be identified with the divisor corresponding to the point $(-2,-3,0)$. The 'top' 


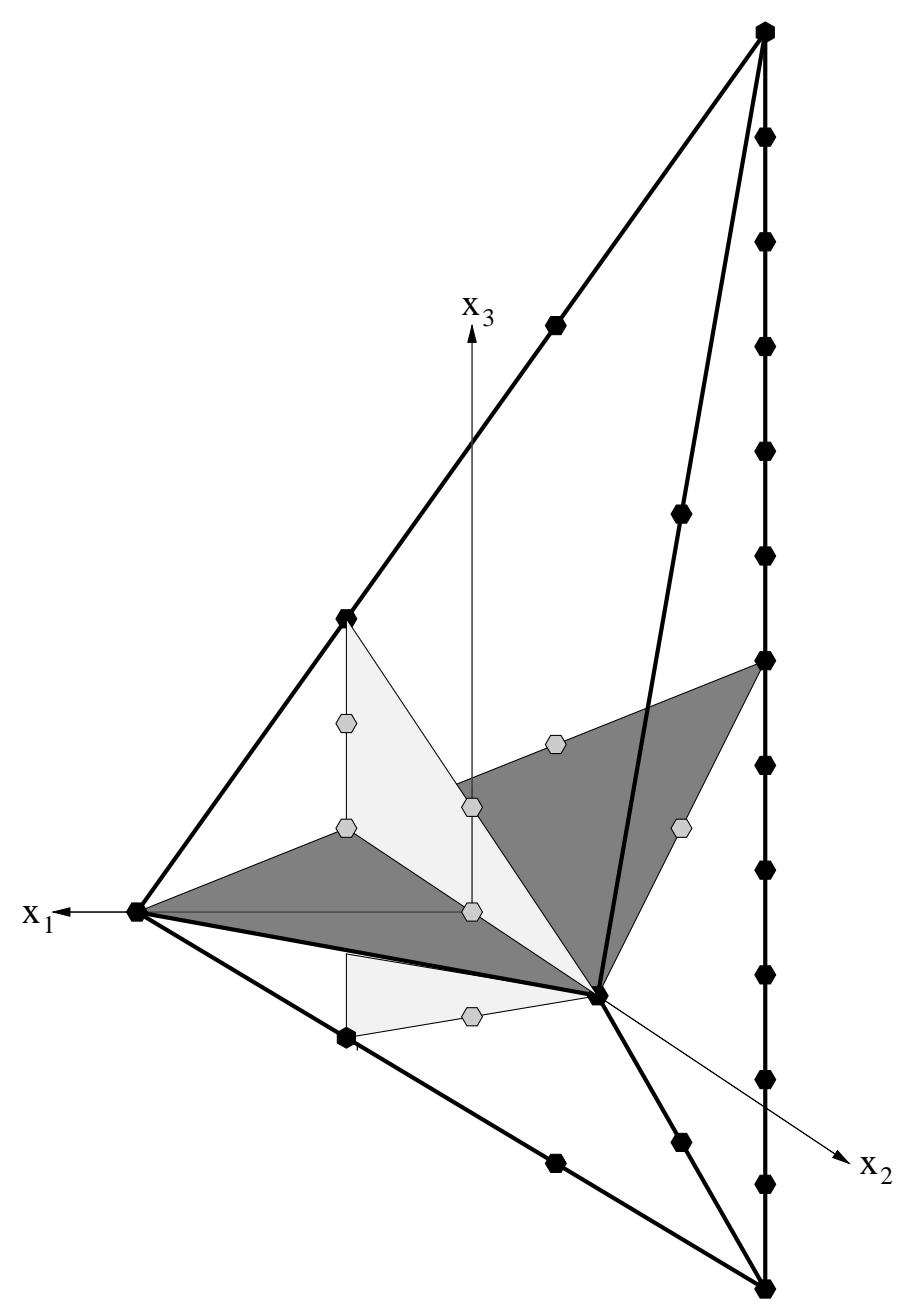

Figure 1: The reflexive polyhedron that contains both the Weierstrass and the new SO(32) triangle.

and the 'bottom' correspond to extended Dynkin diagrams of $E_{8}$, the extension points being the points $(-2,-3,1)$ and $(-2,-3,-1)$ above and below the 'section point', respectively.

In the second case our triangle lies in the plane $x_{1}=0$ and is given as the convex hull of the points

$$
(0,1,0), \quad(0,-1,-2), \text { and }(0,-1,2) \text {. }
$$

This triangle is dual to the Newton polyhedron of $\mathbb{P}_{1,1,2}[4]$. Now the $K 3$ polyhedron is split in an asymmetric way: On one side we have just a single point, the corresponding divisor being a smooth fiber, whereas on the other side we have 17 relevant points forming the extended Dynkin diagram of $S O(32)$. Here we have two different sections, determined by 
$(0,-1,-2)$ and $(0,-1,2)$, respectively. This is in agreement with the assertion in 24] that a fibration giving an $S O(32)$ string should have two distinct sections. Again we take the extension point to be the point adjacent to one of the 'section points'. Compactification of F-theory on the corresponding $K 3$ surface with the fibers corresponding to the Dynkin diagram blown down should be dual to the $S O(32)$ heterotic string on a $T^{2}$ with no Wilson lines on. It is easily checked by considering the dual polyhedron $\Delta$ that the complex structure of this model depends on two free parameters.

The two different fibration structures that can be read off from our polyhedron $\Delta^{*}$ are related to the fact [9] that the Picard lattice $\Gamma_{1,17}$ of the $K 3$ allows two different decompositions as $\Gamma_{1,1} \oplus \Gamma_{16}$.

\section{Lower dimensional compactifications and large gauge groups}

In this section we will give examples of compactifications of F-theory to six and four dimensions such that the polyhedron of the previous section encodes K3 fibers of the corresponding compactification manifolds. Let us start with considering a Calabi-Yau threefold in the family that is mirror dual to $\mathbb{P}_{1,1,12,28,42}[84]$ (this means that $\Delta_{C Y}^{*}$ will be the Newton polyhedron of $\left.\mathbb{P}_{1,1,12,28,42}[84]\right)$. In suitable coordinates the vertices of $\Delta_{C Y}^{*}$ are given by

$$
(1,0,0,0), \quad(0,1,0,0), \quad(-2,-3,-42,-6), \quad(-2,-3,42,-6), \quad(-2,-3,0,1) .
$$

In these coordinates it is easy to see that there are two $K 3$ polyhedra in $\Delta_{C Y}^{*}$. One of them lies in the hyperplane $x_{3}=0$. It corresponds to a 'trivial top' with an $E_{8}$ bottom and will not be considered further. The other one lies in the hyperplane $x_{4}=0$. Its vertices are

$$
(1,0,0,0), \quad(0,1,0,0), \quad(-2,-3,-6,0), \quad(-2,-3,6,0) .
$$

This is just the polyhedron $\Delta_{K 3}^{*}$ we discussed in the previous section. The two different elliptic fibration structures in our polyhedron $\Delta_{K 3}^{*}$ carry over to $\Delta_{C Y}^{*}$. As explained in [25], the fan for the toric variety describing the base of the fibration is determined by projecting the polyhedron along the fiber (more precisely: by considering equivalence classes of points differing only by vectors lying in the plane of the fiber). In the present case, this projection just amounts to throwing away the second and third coordinate of each point. Figure 2 shows the image of $\Delta_{C Y}^{*}$ under this projection and the resulting fan. 


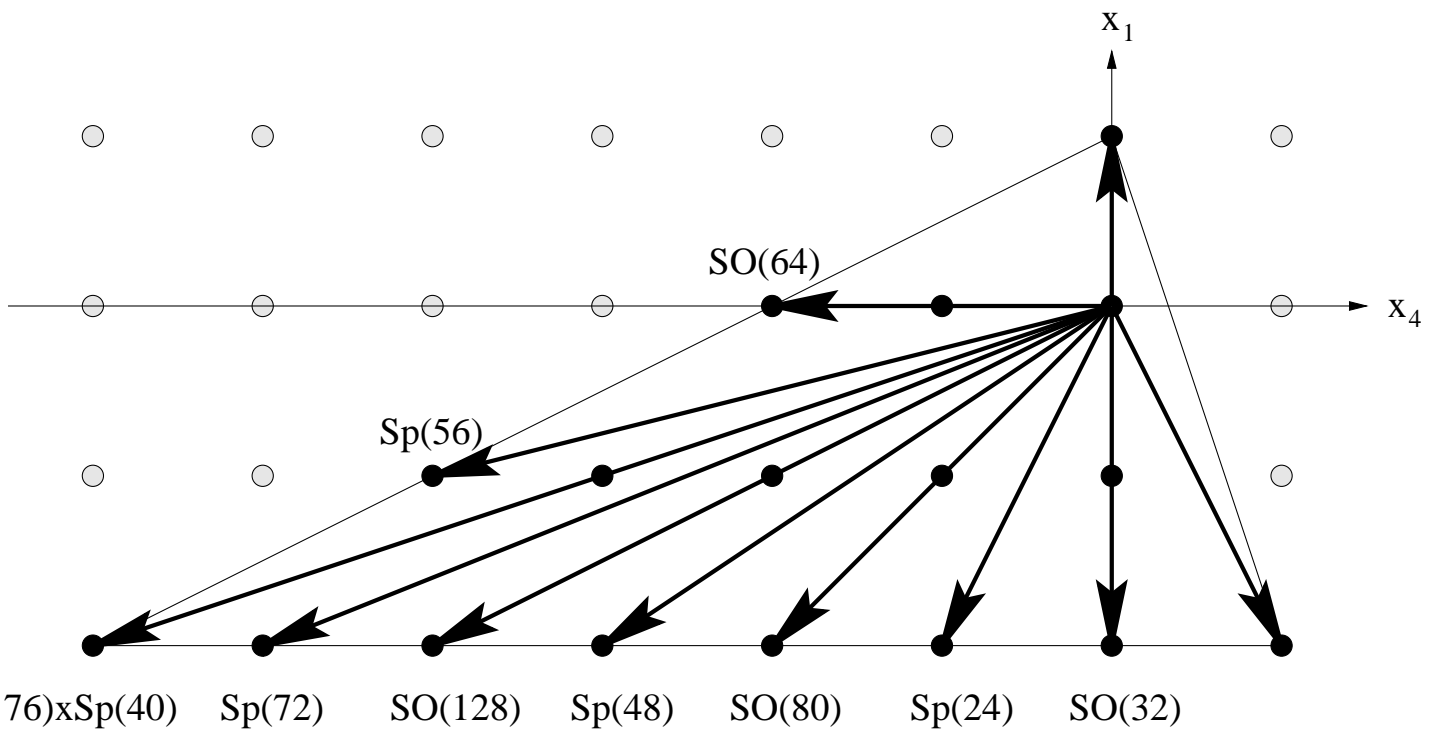

Figure 2: The fan of the base of the $S O(32)$-fibration for the threefold. The rays give rise to the indicated groups.

The elliptic fiber can degenerate along the curves in the base space determined by the toric divisors. The way it degenerates can be determined by considering the preimage of the projection. For the points $\left(x_{1}, x_{4}\right)=(1,0)$ and $(-2,1)$ respectively the preimage consists of only one point, so the corresponding fibers are just smooth elliptic curves. The other divisors in the base space lead to enhanced gauge groups as indicated. The preimages of the corresponding rays are 'tops', where we define a 'top' to be a three dimensional lattice polyhedron with one facet containing the origin and the other facets at integer distance one from the origin. This definition implies that the facet containing the origin is a reflexive polygon. The relevant points of the 'top' without the facet containing the origin form the extended Dynkin diagram of the gauge group. Note that this generalises the concept of a 'top' as a half of a reflexive polyhedron as formulated in [15], since most of the tops we will encounter cannot be completed to a reflexive polyhedron. For the $S O(\cdot)$ groups the 'tops' look similar to the right part of Figure 1, but with more points along the long line. As an example for an ' $S p(\cdot)$ top' Figure 3 shows the 'top' for $S p(24)$.

There are two types of subtleties that can arise from the fact that we interpret points as points of $\Delta_{C Y}^{*}$ on the one hand and as points of a 'top' on the other hand, both related to the fact that they need not correspond to the same number of divisors in each picture. The first of these subtleties occurs when a point that corresponds to precisely one divisor in the Calabi- 


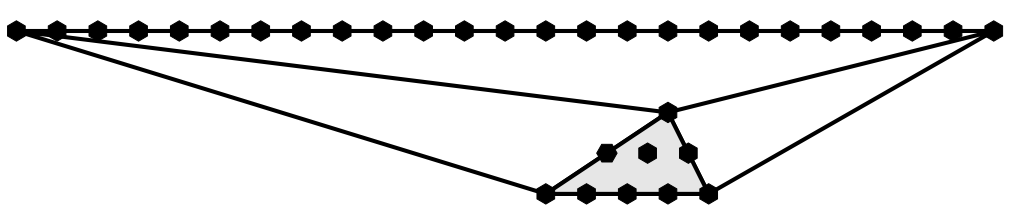

Figure 3: The 'top' for the group $S p(24)$. There are 25 points in a straight line which form the extended Dynkin diagram of the group.

Yau space corresponds to more than one divisor in the top. In this case several divisors that form part of an ADE pattern are identified globally; the corresponding monodromy leads to a non-simply laced gauge group. This is how we get the $S p(\cdot)$ groups. The other special case is when points that are irrelevant in the context of the 'top' turn out to be relevant for the Calabi-Yau space, as discussed in [17]. This happens for the point $(-2,-6)$ in the base. The preimage of the corresponding ray looks like the 'top' for $S O(176)$, but after identifying the points that are relevant in the context of the 'top' with the corresponding Dynkin diagram, we find that there are still points that are relevant in the context of the whole Calabi-Yau space. These points, by themselves, form an $S p(40)$ 'top'. Since in this case the extra points are relevant, the total contribution from the preimage of this ray is $S O(176) \times S p(40)$.

Proceeding similarly with what is possibly the 'largest' fourfold polyhedron, the Newton polyhedron of $\mathbb{P}_{1,1,84,516,1204,1806}[3612]$, we may choose coordinates such that the vertices of $\Delta_{\text {fourfold }}^{*}$ are given by

$$
\begin{gathered}
(1,0,0,0,0), \quad(0,1,0,0,0), \quad(-2,-3,3606,-6,-6), \quad(-2,-3,-6,37,-6), \\
(-2,-3,-6,-6,1), \quad(-2,-3,-6,-6,-6) .
\end{gathered}
$$

The $E_{8} \times E_{8}$ or $S O(32)$ polyhedron lies in the plane $x_{4}=x_{5}=0$ and the $S O(32)$ triangle lies in the $x_{2} x_{3}$-plane. The base of the fibration is therefore determined by projecting out $x_{2}$ and $x_{3}$. This results in a tetrahedron $T$ with vertices

$$
(1,0,0), \quad(-2,-6,-6), \quad(-2,37,-6), \quad(-2,-6,1) .
$$

The preimages of its vertices are single points, the exception being the vertex $(-2,-6,-6)$ whose preimage consists of 3613 lattice points in a row. Together with the preimage of $(-1,-3,-3)$ which lies on the same ray, this gives the 'top' for the group $S O(7232)$. The points that are irrelevant in the context of this top are relevant in the context of the fourfold, so we get an additional group factor of $S p(1804)$. The preimages of other points along the 
boundary of $T$ are always lines whose numbers of points can be determined by linearity; e.g. the preimage of

$$
(-2,0,0)=\frac{6}{7}(-2,-6,1)+\frac{6}{43}(-2,37,-6)+\frac{1}{7 \times 43}(-2,-6,-6)
$$

consists of

$$
\frac{6}{7} \times 1+\frac{6}{43} \times 1+\frac{1}{7 \times 43} \times 3613=13
$$

points giving rise to the unbroken $S O(32)$. In general $k$ points in a row give rise to $S O(2 k+6)$ or $S p(k-1)$, depending on whether the point in the boundary of $T$ is divisible by two or not. If a point that leads to an $S O(\cdot)$ group lies on an edge of $T$, then there is an additional factor of $S p\left(\frac{k-5}{2}\right)$. Altogether we get a gauge group of rank 302,896 consisting of 251 nontrivial simple factors, the largest of them being $S O(7232)$ and $S p(3528)$ and the smallest being $S O(32)$ and $S p(24)$. The full list of factors is given in the appendix. It is interesting that the $S O(32)$ decomposition seems to produce groups of larger rank than the $E_{8} \times E_{8}$ decomposition. For the fourfold we have been considering the $E_{8} \times E_{8}$ decomposition leads to the group [17] $E_{8}^{2561} \times F_{4}^{7576} \times G_{2}^{20168} \times S U(2)^{30200}$ of rank 121, 328 while for the $S O(32)$ decomposition the rank is larger. The reason for this is that the number of divisors in the base is smaller in the $S O(32)$ case because here we project along the direction along which the extension of the polytope is largest.

Acknowledgements: We would like to thank Eugene Perevalov and Govindan Rajesh for useful discussions. This work is supported by the Austrian 'Fonds zur Förderung der wissenschaftlichen Forschung' (Schrödinger fellowship J012328-PHY), by NSF grant PHY9511632 and the Robert A. Welch Foundation. 


\section{References}

[1] C. Vafa, Evidence for F-Theory, Nucl. Phys. B469 (1996) 403, hep-th/9602022.

[2] C. Hull and P. Townsend, Unity of Superstring Dualities, Nucl. Phys. B438 (1995) 109, hepth/9410167.

[3] E. Witten, String Theory Dynamics in Various Dimensions, Nucl. Phys. B443 (1995) 85, hep-th/9503124.

[4] D.R. Morrison and C. Vafa, Compactifications of F-Theory on Calabi-Yau Threefolds - I, Nucl. Phys. B473 (1996) 74, hep-th/9602114.

[5] D.R. Morrison and C. Vafa, Compactifications of F-Theory on Calabi-Yau Threefolds - II, Nucl. Phys. B476 (1996) 437, hep-th/9603161.

[6] E. Witten, Small Instantons in String Theory, Nucl. Phys. B460 (1996) 541, hep-th/9511030.

[7] N. Seiberg, E. Witten, Comments on String Dynamics in Six-Dimensions, Nucl. Phys. B471 (1996) 121, hep-th/9603003.

[8] R. Friedman, J. Morgan, E. Witten, Vector Bundles and F Theory, hep-th/9701162.

[9] P.S. Aspinwall, D.R. Morrison, Point-like Instantons on K3 Orbifolds, hep-th/9705104.

[10] K. Intriligator, RG Fixed Points in Six Dimensions via Branes at Orbifold Singularities, hepth/9702038.

[11] J.D. Blum, K. Intriligator, Consistency Conditions for Branes at Orbifold Singularities, hepth/9705030.

[12] J.D. Blum, K. Intriligator, New Phases of String Theory and 6d RG Fixed Points via Branes at Orbifold Singularities, hep-th/9705044.

[13] A. Klemm, P. Mayr, Strong Coupling Singularities and Non-Abelian Gauge Symmetries in N=2 String Theory, hep-th/9601014.

[14] S. Katz, D.R. Morrison, M.R. Plesser, Enhanced Gauge Symmetry in Type II String Theory, Nucl. Phys. B477 (1996) 105 hep-th/9601108.

[15] P. Candelas, A.Font, Duality Between the Webs of Heterotic and Type II Vacua, hepth/9603170, revised version (March 1997).

[16] E. Perevalov, H. Skarke, Enhanced Gauge Symmetry in Type II and F-Theory Compactifications: Dynkin Diagrams from Polyhedra, hep-th/9704129. 
[17] P. Candelas, E. Perevalov and G. Rajesh, Toric Geometry and Enhanced Gauge Symmetry of F-Theory/Heterotic Vacua, hep-th/9704097.

[18] D. Cox, The homogeneous coordinate ring of a toric variety, J. Alg. Geom. 4 (1995) 17, alg-geom/9210008.

[19] V.V. Batyrev, Dual polyhedra and mirror symmetry for Calabi-Yau hypersurfaces in toric varieties, J. Alg. Geom. 3 (1994) 493, alg-geom/9310003.

[20] P. Candelas, X. de la Ossa, S. Katz, Mirror Symmetry for Calabi-Yau Hypersurfaces in Weighted $\mathbb{P}_{4}$ and Extensions of Landau-Ginzburg Theory, Nucl. Phys. B450 (1995) 267, hep-th/9412117.

[21] A. Avram, M. Mandelberg, M. Kreuzer and H. Skarke, Searching for K3 Fibrations, hepth/9610154.

[22] M. Kreuzer, H. Skarke, On the Classification of Reflexive polyhedra, hep-th/9512204.

[23] H. Skarke, Weight Systems for Toric Calabi-Yau Varieties and Reflexivity of Newton Polyhedra, Mod. Phys. Lett. A11 (1996) 1637, alg-geom/9603007.

[24] P.S. Aspinwall and M. Gross, The SO(32) Heterotic String on a K3 Surface, Phys. Lett. B387 (1996) 735 , hep-th/9605131.

[25] M. Kreuzer and H. Skarke, Calabi-Yau Fourfolds and Toric Fibrations, hep-th/9701175. 


\section{Appendix: The groups that arise from the toric divisors of the base}

\begin{tabular}{|c|c|c|c|c|c|c|}
\hline $\mathrm{Sp}[0]$ & $\operatorname{Sp}[168]$ & $\mathrm{Sp}[336]$ & $\mathrm{Sp}[504]$ & $\operatorname{Sp}[72]$ & & $\mathrm{Sp}[156]$ \\
\hline $\mathrm{Sp}[672]$ & $\mathrm{Sp}[240]$ & $\mathrm{Sp}[324]$ & $\mathrm{Sp}[840]$ & $\mathrm{Sp}[408]$ & & $\mathrm{Sp}[492]$ \\
\hline $\mathrm{Sp}[1008]$ & $\operatorname{Sp}[56]$ & $\mathrm{Sp}[576]$ & $\mathrm{Sp}[140]$ & $\mathrm{Sp}[144]$ & & $\mathrm{Sp}[660]$ \\
\hline $\operatorname{Sp}[1176]$ & $\mathrm{Sp}[224]$ & $\mathrm{Sp}[744]$ & $\mathrm{Sp}[308]$ & $\mathrm{Sp}[312]$ & & $\mathrm{Sp}[828]$ \\
\hline $\mathrm{Sp}[1344]$ & $\mathrm{Sp}[392]$ & $\mathrm{Sp}[912]$ & $\mathrm{Sp}[476]$ & $\mathrm{Sp}[480]$ & & $\mathrm{Sp}[996]$ \\
\hline $\operatorname{Sp}[1512]$ & $\mathrm{SO}[176] \mathrm{Sp}[40]$ & $\operatorname{Sp}[48]$ & $\operatorname{Sp}[560]$ & $\operatorname{Sp}[1080]$ & $\mathrm{SO}[512]$ & 2] $\mathrm{Sp}[124]$ \\
\hline $\mathrm{Sp}[132]$ & $\mathrm{Sp}[644]$ & $\mathrm{Sp}[648]$ & $\operatorname{Sp}[1164]$ & $\mathrm{Sp}[1680]$ & $\mathrm{SO}[848]$ & 8] $\mathrm{Sp}[208]$ \\
\hline $\mathrm{Sp}[216]$ & $\mathrm{Sp}[728]$ & Sp[1248], & $\mathrm{SO}[1184] \mathrm{Sp}[292]$, & $\mathrm{Sp}[300]$, & & $\mathrm{Sp}[812]$ \\
\hline $\operatorname{Sp}[816]$ & $\mathrm{Sp}[1332]$ & $\mathrm{Sp}[1848]$ & $\mathrm{SO}[1520] \mathrm{Sp}[376]$ & $\mathrm{Sp}[384]$ & & $\mathrm{Sp}[896]$ \\
\hline $\operatorname{Sp}[1416]$ & $\mathrm{SO}[1856] \mathrm{Sp}[460]$ & $\mathrm{Sp}[468]$ & $\mathrm{Sp}[980]$ & $\mathrm{Sp}[984]$ & & $\mathrm{Sp}[1500]$ \\
\hline $\mathrm{Sp}[2016]$ & $\mathrm{SO}[128]$ & $\mathrm{SO}[2192] \mathrm{Sp}[544]$ & $\mathrm{Sp}[552]$ & $\mathrm{Sp}[1064]$ & & $\operatorname{Sp}[1584]$ \\
\hline $\mathrm{Sp}[112]$ & $\mathrm{SO}[464]$ & $\mathrm{Sp}[120]$ & $\mathrm{SO}[2528] \mathrm{Sp}[628]$ & $\mathrm{Sp}[636]$ & & $\operatorname{Sp}[1148]$ \\
\hline $\operatorname{Sp}[1152]$ & $\operatorname{Sp}[1668]$ & $\operatorname{Sp}[2184]$ & $\mathrm{SO}[800]$ & $\mathrm{SO}[2864] \mathrm{Sp}[712]$ & & $\mathrm{Sp}[720]$ \\
\hline $\operatorname{Sp}[1232]$ & $\operatorname{Sp}[1752]$ & $\mathrm{Sp}[280]$ & $\mathrm{SO}[1136]$ & $\mathrm{Sp}[288]$ & $\mathrm{SO}[3200]$ & 0] $\mathrm{Sp}[796]$ \\
\hline $\mathrm{Sp}[804]$ & $\operatorname{Sp}[1316]$ & $\operatorname{Sp}[1320]$ & $\operatorname{Sp}[1836]$ & $\mathrm{Sp}[2352]$ & & $\mathrm{SO}[1472]$ \\
\hline $\mathrm{SO}[3536] \mathrm{Sp}[880]$ & $\mathrm{Sp}[888]$ & $\mathrm{Sp}[1400]$ & $\operatorname{Sp}[1920]$ & $\mathrm{Sp}[448]$ & & $\mathrm{SO}[1808]$ \\
\hline $\operatorname{Sp}[456]$ & $\mathrm{SO}[3872] \mathrm{Sp}[964]$ & $\mathrm{Sp}[972]$ & $\mathrm{Sp}[1484]$ & $\mathrm{Sp}[1488]$ & & $\operatorname{Sp}[2004]$ \\
\hline $\mathrm{Sp}[2520]$ & $\mathrm{SO}[64]$ & $\mathrm{SO}[80]$ & $\mathrm{Sp}[24]$ & $\mathrm{SO}[2144]$ & $\mathrm{SO}[4208]$ & ] $\mathrm{Sp}[1048]$ \\
\hline $\operatorname{Sp}[1056]$ & $\mathrm{Sp}[1568]$ & $\mathrm{Sp}[2088]$ & $\mathrm{SO}[400]$ & $\mathrm{SO}[416]$ & & $\mathrm{Sp}[108]$ \\
\hline $\operatorname{Sp}[616]$ & $\mathrm{SO}[2480]$ & $\mathrm{Sp}[624]$ & $\mathrm{SO}[4544] \mathrm{Sp}[1132]$ & $\mathrm{Sp}[1140]$ & & $\operatorname{Sp}[1652]$ \\
\hline $\operatorname{Sp}[1656]$ & $\mathrm{Sp}[2172]$ & $\mathrm{Sp}[2688]$ & $\mathrm{SO}[736]$ & $\mathrm{SO}[752]$ & & $\mathrm{Sp}[192]$ \\
\hline $\mathrm{SO}[2816]$ & $\mathrm{SO}[4880] \mathrm{Sp}[1216]$ & $\mathrm{Sp}[1224]$ & $\operatorname{Sp}[1736]$ & $\mathrm{Sp}[2256]$ & & $\mathrm{SO}[1072]$ \\
\hline $\mathrm{SO}[1088]$ & $\mathrm{Sp}[276]$ & $\mathrm{Sp}[784]$ & $\mathrm{SO}[3152]$, & $\mathrm{Sp}[792]$ & $\mathrm{SO}[5216]$ & Sp[1300] \\
\hline $\mathrm{Sp}[1308]$ & $\operatorname{Sp}[1820]$ & $\operatorname{Sp}[1824]$ & $\mathrm{Sp}[2340]$ & $\mathrm{Sp}[2856]$ & & $\mathrm{SO}[1408]$ \\
\hline $\mathrm{SO}[1424]$ & $\mathrm{Sp}[360]$ & $\mathrm{SO}[3488]$ & $\mathrm{SO}[5552] \mathrm{Sp}[1384]$ & $\mathrm{Sp}[1392]$ & & $\operatorname{Sp}[1904]$ \\
\hline $\mathrm{Sp}[2424]$ & $\mathrm{SO}[1744]$ & $\mathrm{SO}[1760]$ & $\mathrm{Sp}[444]$ & $\mathrm{Sp}[952]$ & & $\mathrm{SO}[3824]$ \\
\hline $\mathrm{Sp}[960]$ & $\mathrm{SO}[5888] \mathrm{Sp}[1468]$ & $\operatorname{Sp}[1476]$ & $\mathrm{Sp}[1988]$ & Sp[1992] & & $\mathrm{Sp}[2508]$ \\
\hline $\operatorname{Sp}[3024]$ & $\mathrm{Sp}[0]$ & $\mathrm{SO}[32]$ & $\mathrm{SO}[2080]$ & $\mathrm{SO}[2096]$ & & $\mathrm{Sp}[528]$ \\
\hline $\mathrm{SO}[4160]$ & $\mathrm{SO}[6224] \mathrm{Sp}[1552]$ & $\operatorname{Sp}[1560]$ & $\mathrm{Sp}[2072]$, & $\mathrm{Sp}[2592]$ & & $\mathrm{SO}[352]$, \\
\hline $\mathrm{SO}[368]$ & $\mathrm{Sp}[96]$ & $\mathrm{SO}[2416] \mathrm{Sp}[600]$ & $\mathrm{SO}[2432]$ & $\mathrm{Sp}[612]$ & & $\operatorname{Sp}[1120]$ \\
\hline $\mathrm{SO}[4496]$ & $\operatorname{Sp}[1128]$ & $\mathrm{SO}[6560] \mathrm{Sp}[1636]$ & $\operatorname{Sp}[1644]$ & $\mathrm{Sp}[2156]$ & & $\operatorname{Sp}[2160]$ \\
\hline $\operatorname{Sp}[2676]$ & $\mathrm{Sp}[3192]$ & $\mathrm{SO}[704]$ & $\mathrm{Sp}[688]$ & $\mathrm{SO}[2768]$ & & $\mathrm{Sp}[696]$ \\
\hline $\mathrm{SO}[4832]$ & $\mathrm{SO}[6896] \mathrm{Sp}[1720]$ & $\mathrm{Sp}[1728]$ & $\mathrm{Sp}[2240]$ & $\operatorname{Sp}[2760]$ & $\mathrm{SO}[1040]$ & 0] $\mathrm{Sp}[256]$, \\
\hline $\operatorname{Sp}[264]$ & $\mathrm{SO}[3104] \mathrm{Sp}[772]$ & $\mathrm{Sp}[780]$ & $\mathrm{SO}[5168] \mathrm{Sp}[1288]$ & $\mathrm{Sp}[1296]$ & $\mathrm{SO}[7232]$ & Sp[1804] \\
\hline $\mathrm{Sp}[1812]$ & $\mathrm{Sp}[2324]$ & $\mathrm{Sp}[2328]$ & $\operatorname{Sp}[2844]$ & $\mathrm{Sp}[3360]$ & & $\mathrm{Sp}[344]$ \\
\hline $\mathrm{Sp}[860]$ & $\mathrm{Sp}[864]$ & $\mathrm{Sp}[1376]$ & $\mathrm{Sp}[1892]$ & $\mathrm{Sp}[1896]$ & & $\mathrm{Sp}[2408]$ \\
\hline $\mathrm{Sp}[2928]$ & $\mathrm{Sp}[432]$ & $\mathrm{Sp}[948]$ & $\operatorname{Sp}[1464]$ & $\mathrm{Sp}[1980]$ & & $\operatorname{Sp}[2496]$ \\
\hline $\operatorname{Sp}[3012]$, & $\operatorname{Sp}[3528]$ & $\operatorname{Sp}[0]$, & $\operatorname{Sp}[1032]$ & $\operatorname{Sp}[2064]$ & & $\mathrm{Sp}[3096]$ \\
\hline
\end{tabular}

